livraisons

d'Histoire

de l'Architecture

\section{Livraisons de l'histoire de l'architecture}

25 | 2013

Architectures médiévales : types, matières et formes

\title{
Les horloges publiques : un aspect méconnu du mécénat du duc Jean de Berry
}

Public clocks: a little known aspect of patronage of duke Jean de Berry

Die öffentlichen Turmuhren : ein unbekannter Aspekt des Mäzenatentums von Jean Herzog von Berry

\section{Thomas Rapin}

\section{(2) OpenEdition}

Journals

Édition électronique

URL : http://journals.openedition.org/lha/539

DOI : $10.4000 /$ /ha. 539

ISSN : 1960-5994

Éditeur

Association Livraisons d'histoire de l'architecture - LHA

Édition imprimée

Date de publication : 10 juin 2013

Pagination : $97-108$

ISSN : 1627-4970

Référence électronique

Thomas Rapin, "Les horloges publiques : un aspect méconnu du mécénat du duc Jean de Berry »,

Livraisons de l'histoire de l'architecture [En ligne], 25 | 2013, mis en ligne le 10 juin 2015, consulté le 30 avril 2019. URL : http://journals.openedition.org//ha/539 ; DOI : 10.4000//ha.539

Ce document a été généré automatiquement le 30 avril 2019

Tous droits réservés à l'Association LHA 


\section{Les horloges publiques : un aspect méconnu du mécénat du duc Jean de Berry}

Public clocks: a little known aspect of patronage of duke Jean de Berry

Die öffentlichen Turmuhren : ein unbekannter Aspekt des Mäzenatentums von Jean Herzog von Berry

\section{Thomas Rapin}

Je me puis bien comparer a l'orloge, Car quant Amours, qui en mon coer se loge, M'i fait penser et mettre y mon estude,

G'i aperçoi une similitude Jean Froissart, L'Orloge Amoureus (v. 1-4)

\section{L'horlogerie au service des puissants}

1 Entre 1385 et 1389, Jean de France, duc de Berry, d'Auvergne et comte de Poitou (1340-1416), fit édifier aux abords de son palais de Poitiers un beffroi haut de plus de 41 mètres communément appelé le "Gros horloge ». Quatre siècles durant, sa sonnerie annonça les heures du jour et de la nuit aux poitevins ${ }^{1}$. Avant d'aborder dans le détail les relations qu'entretenait le prince avec l'horlogerie, arrêtons-nous un instant sur le caractère avant tout symbolique du programme. Certes, le mécène n'était pas le premier à doter une ville d'un semblable édifice ; le phénomène avait commencé dès l'aube du XIVe siècle. Il n'en demeure pas moins vrai que l'horlogerie - ou plutôt la possession et l'utilisation d'instruments de mesure du temps - resta longtemps l'attribut des puissants avant d'être peu à peu diffusée auprès des populations. Loin d'un simple équipement urbain, il faut voir dans ce don - qui engagea l'implication personnelle du prince - une concession du seigneur à sa bonne ville. 
2 Le fait que l'horlogerie était un art retenu dans les sphères du pouvoir tenait à deux raisons essentielles. Tout d'abord seuls les grands seigneurs étaient en mesure de réunir les talents nécessaires à leur réalisation: ceux des scientifiques (mathématiciens et astronomes) d'une part et ceux des plus habiles serruriers et ferronniers d'autre part. De telles compétences étaient rares. Aucun statut ne définissait encore le métier et aucun apprentissage n'y préparait. En outre, l'usage de tels instruments restait à la discrétion d'initiés ayant accès aux savoirs des anciens grecs et des arabes. Par exemple, en 1391, le roi Jean d'Aragon fit présent d'un astrolabe, d'une mappemonde et d'un almanach au comte de Foix. L'expédition de ces objets se fit accompagnée d'un savant chargé d'en expliquer le fonctionnement à ce seigneur. Par ailleurs, l'usage d'horloges n'avait d'intérêt que dans certains milieux. Il fixait l'exercice de la liturgie des grandes communautés religieuses de même que le cadre protocolaire des grandes cours seigneuriales dont on connaît le goût croissant pour les livres d'heures à la fin du XIV siècle. Christine de Pisan témoigne de la stricte discipline horaire à laquelle s'astreignait Charles V. Du reste, d'aucuns prétendent qu'il fut le premier roi à faire sonner les heures de la journée par les cloches de Paris².

On comprend mieux dès lors l'importance de la diffusion de ce savoir vers les places publiques qui marque nécessairement la montée en puissance des pouvoirs urbains. À cet égard, il est significatif de constater que les premiers beffrois équipèrent les villes d'Italie et celles de l'Empire. En France, elle accompagne la progressive émergence des « bonnes villes » à partir du milieu du XIV ${ }^{e}$ siècle. La division de la journée partagée par l'ensemble des citadins s'imposait pour organiser les formes de plus en plus complexes du commerce, de l'artisanat, de la finance, mais aussi l'exercice de la justice. Dans la première moitié du $\mathrm{XIV}^{\mathrm{e}}$ siècle à Valenciennes, l'activité de la halle aux draps était réglée par une horloge ${ }^{3}$. En 1395, les habitants de Beaune édifièrent une horloge "utile » aux juges et aux officiers ducaux. C'est donc bien ces nouveaux acteurs - relais essentiels de l'état moderne en devenir - qui entrèrent à leur tour dans le cercle de plus en plus large des utilisateurs d'horloges. Ce que le seigneur concédait, il pouvait le reprendre comme par exemple à l'encontre d'une ville rebelle. Ce fut le cas pour le beffroi de Rouen après la Harelle (1382) ${ }^{4}$ ou le jacquemart de Kortrijk démonté par Philippe le Hardi après la victoire de Roosebeke (1382) et rapporté jusqu'à Dijon tel un trophée ${ }^{5}$.

4 Dans l'Europe du XIV ${ }^{e}$ siècle, nombreux sont les exemples de ce mécénat si particulier. Car il s'agit bien d'un mécénat. Au même titre que la promotion de l'art, la culture et les sciences, les puissants entendaient contrôler à leur profit la mesure du temps qu'ils considéraient comme leur revenant de droit et dans laquelle ils voyaient un moyen parmi d'autres d'asseoir leur légitimité d'essence divine. Curieusement les horloges commandées par Jean de Berry sont restées un sujet boudé par ceux, nombreux, qui se sont penchés sur son mécénat. Les auteurs de la célèbre monographie Travaux d'art réalisés pour Jean de France, n'en disent mot. Il faut attendre vingt années pour que l'un d'eux consacre à ce thème la place qu'il méritait dans une courte contribution qui resta malheureusement dans l'oubli'.

\section{Les horlogers de Jean de Berry}

5 C'est principalement dans le nord du royaume et en terre d'Empire que les princes recrutaient leurs «orlogeurs ». L'essor de l'horlogerie monumentale y fut précoce et ses techniciens solidement formés. Pour l'horloge du palais de la Cité, Charles V fit venir 
Henri de Vic (entre Meuse et Rhin)1 ${ }^{1}$ En Bourgogne, Philippe le Hardi recruta Jean d'Allemagne et Hue de Boulogne. Marguerite de Flandre prit à son service le Lillois Pierre de Daimleville. On connaît trois horlogers entrés au service de Jean de Berry: Jean de Wissembourg (au nord de l'Alsace), un certain Hanry et Pierre Merlin². Peu nombreux et circulant au grès des commandes, beaucoup d'horlogers connurent plusieurs protecteurs comme le sénonais Pierre de Sainte-Béate qui travailla pour Charles $V$ et les papes à Avignon ou Pierre Merlin pour Charles VI, le duc d'Anjou et le duc de Berry. La rareté de ces talents les fit entrer par la grande porte dans les hôtels des rois et des princes au même titre que les Raymond Du Temple, André Beauneuveu ou Guy de Dampmartin. Pierre de Daimleville, par exemple, recevait de la duchesse des draps des officiers et une livrée. Un de ses confrères faisait partie des valets de chambre de Philippe le Hardi.

6 Derrière le terme "horloger " se cachent des situations très différentes. Par exemple, nous ne trouvons pas dans l'entourage de Jean de Berry d'horlogers instruits des sciences de la médecine, des mathématiques et de l'astronomie, comme pouvaient l'être les célèbres Jacques et Jean Dondi de Padoue, les créateurs de l'Astrarium - chef d'œuvre resté longtemps inégalé capable de reproduire «la première analogie du mouvement des planètes" - ou encore le chanoine parisien Jean Fusoris qui conçut l'horloge astronomique de Bourges, horloge qu'il ne faut pas confondre avec celle installée par Jean de Berry dans cette même église. Ces savants étaient des lettrés qui laissèrent à la postérité plusieurs traités techniques ${ }^{3}$. C'est à côté de ces grandes figures tutélaires que nous trouvons nos fabricants d'horloges publiques. Comparée aux cadrans astronomiques, aux mobiles lunaires ou zodiacaux, les horloges publiques affichent la plus grande simplicité. Elles indiquaient l'heure aux quidams. Du reste ajoutons que les horloges commandées par Jean de Berry étaient même dépourvues de cadran. Elles étaient donc conçues pour être entendues davantage que vues.

7 Qui étaient ces horlogers? Formés de manière plus modeste et probablement empirique, ils n'en déméritèrent pas moins et furent reconnus pour leur parfaite maîtrise technique. Par exemple, Pierre de Sainte-Béate, l'horloger de Charles V, reçut la commande d'une horloge portative véritable gageure technologique pour l'époque. Un marché passé pour le compte de Margueritte de Flandre en 1379 illustre davantage l'ordinaire d'un horloger à la cour. Il stipule que le Lillois Pierre de Daimleville fut chargé de réaliser et d'installer une horloge pour le clocher du château de Nieppe en lieu et place d'une plus ancienne. Le prestataire fournissait la matière d'œuvre et perçut 40 francs d'or pour la livraison de près de $150 \mathrm{~kg}$ de fer. L'administration de la duchesse s'engageait par ailleurs à verser un supplément si la quantité de métal s'avérait insuffisante. Durant l'installation, Pierre de Daimleville pouvait loger sur place « sans autre gage ». Enfin comme il était d'usage dans ce genre de contrat, l'horloger engageait sa responsabilité qui supposait qu'il interviendrait à ses frais en cas de défaut de fonctionnement. La tâche de l'horloger ne prenait pas fin avec l'installation du mécanisme ; elle commençait même pourrait-on dire. L'entretien et la surveillance étaient fondamentaux car, au même titre que l'architecture, le commanditaire d'une horloge s'engageait sur le très long terme. Rappelons que les mécanismes précédant l'introduction du pendule par Christian Huygens (1657) étaient d'une grande imprécision et devaient être remis à l'heure de manière quasi quotidienne. Il existait à cette fin un personnel chargé de "gouverner» les instruments. Dans de nombreux cas, les gouverneurs d'horloge étaient même logés sur place ${ }^{4}$.

8 Au grès de ces quelques exemples, nous voyons s'esquisser de manière plus précise les contours d'un métier en gestation. Des métiers dirons-nous même car il est important de 
bien distinguer les fonctions de conception, réalisation, installation et de gouvernement qui pouvaient être assurées par des personnages différents.

\section{Les horloges publiques et la politique architecturale de Jean de Berry}

9 L'installation d'horloges publiques dans les villes de France posait un problème de maîtrise d'ouvrage. Les puissantes villes d'Empire et d'Italie pouvaient engager de semblables travaux sur leurs deniers, mais aucune de celles administrées par le prince. $\mathrm{Ni}$ Bourges, ni Poitiers, ni Riom n'avaient les moyens financiers de Padoue ou de Strasbourg capable d'achever sa cathédrale par l'une des plus hautes flèches d'Europe ${ }^{1}$. C'est donc principalement à travers le mécénat de la grande aristocratie que ce phénomène put se développer en France : Charles V au palais de la Cité2 ${ }^{2}$ Charles VI à Lyon et à Nîmes ${ }^{3}$, Louis d'Anjou à la cathédrale Saint-Maurice d'Angers, Philippe le Hardi à Dijon. Au moins huit horloges publiques furent installées à l'instigation de Jean de Berry. Nous traiterons ici uniquement des deux premières pour lesquelles nous disposons d'une documentation suffisante : Bourges (1371-1374) et Poitiers (1385-1389). Pour le reste nous savons qu'en 1387 la ville de Saint-Flour reçut une horloge ${ }^{4}$. Le duc fit installer, avant 1391, une horloge dans une haute tour située au centre de Riom ${ }^{5}$. En 1396, Jean de Berry ordonnait la construction d'une horloge proche des halles de Niort. Pour l'occasion, il accorda aux habitants la somme de $80 £^{6}$. Il fit équiper encore les villes de Mehun-sur-Yèvre (en 1399)7 de Saint-Jean-d'Angely (vers 1400) ${ }^{8}$, de Villefranche-de-Rouergue ${ }^{9}$.

\section{L'Horloge de la cathédrale de Bourges, 1371-1374}

Jean de Berry ne ménagea pas sa peine pour séduire son nouveau duché de Berry brutalement aliéné du domaine royal par le traité de Brétigny. Bien avant d'en faire sa belle capitale, le prince s'appropria l'antique cité : réaménagement des lignes de défense de la Grosse tour, installation de la Chambre des comptes, reconstruction du palais ducal. Mais son premier chantier concerna bien évidement l'église métropolitaine un temps retenue pour lieu d'inhumation. En 1369, il y fonda une messe qui fut rapidement suivie par le réaménagement d'une chapelle (1369-1371). Enfin il fit installer au frontispice de l'édifice une horloge pour les citadins. La première horloge publique commandée par le prince suivait de très peu l'exemple de Charles $\mathrm{V}$ au palais de la Cité. Le prince avait compris toute la portée du programme par lequel il entendait sceller une entente durable avec la puissante église métropolitaine ainsi qu'avec ses paroissiens.

11 Paul Gauchery suppose que l'horloge avait été primitivement installée à la tour nord de la façade et qu'elle fut déplacée au pignon en raison de la reconstruction de cette tour au début du XVIe siècle ${ }^{10}$. L'inscription de la cloche, transcrite par plusieurs érudits, nous révèle la date de la fonte de la cloche : $1372^{11}$. Jean de Berry fit cinq courts séjours à Bourges cette année entre le 24 mars et le 11 décembre. C'est probablement lors d'une de ces visites qu'eut lieu la dédicace. Les comptes de l'Hôtel ducal indiquent qu'en 1371, le prince s'était adjoint les services d'un horloger au nom de Jean de Wissembourg. On engageait également une dépense de 400 francs d'or pour commencer la réalisation du mécanisme $^{12}$. L'ouvrage dut être monté sur place car de Wissembourg logeait chez l'habitant au moins jusqu'en 1373. On sait qu'en 1374, l'administration du duc affectait 
encore à l'horloge de Bourges une somme comprise entre 480 et $500 £$ unique subvention ducale accordée cette année à la construction outre le chantier de Nonette ${ }^{13}$.

\section{Le Gros Horloge de Poitiers, 1385-1389}

12 Le programme du Gros horloge de Poitiers s'inscrivait dans un contexte bien différent. Le mécène et ses frères avaient multiplié de semblables programmes dans le royaume. Les expériences s'étaient croisées, les hommes, les modèles, les techniques avaient déjà circulé. Contrairement au Bourges des années 1370, Poitiers était une ville qui vivait au rythme permanent des chantiers. La commune avait relevé ses remparts et ses tours. Les chantiers du château de Poitiers et de la tour Maubergeon arrivaient à leur terme ; celui du château de Lusignan était bien engagé. La forêt de Coulombiers était exploitée; plusieurs carrières avaient été ouvertes. Les hommes du prince avaient installé leurs ateliers un peu partout dans l'espace urbain à Montierneuf, chez les cordeliers, chez les jacobins et dans plusieurs hôtels ${ }^{14}$. En 1385, Jean de Berry n'était plus le jeune prince tentant de se faire accepter par ses sujets; au faîte de sa puissance, son autorité était alors incontestée. Commanditaire d'une dizaine de chantiers répartis dans l'ensemble de ces domaines et à Paris, le maître d'ouvrage connaissait son apogée. Poitiers devint à ce moment le nouveau centre artistique de l'apanage. Guy de Dampmartin, le général maître des œuvres du duc, vint s'installer dans la cité poitevine afin d'y préparer l'un de ses plus fameux chefs d'œuvre : la reconstruction du pignon sud de la grande salle du palais. Tous les plus grands artistes du prince se retrouvèrent ainsi à Poitiers quelques années avant d'être appelés en Berry et en Auvergne sur les derniers grands chantiers du mécène : la Sainte-Chapelle de Bourges et la chapelle du palais de Riom.

Le cas du Gros horloge de Poitiers est unique dans notre documentation par le nombre de pièces comptables conservées qui permet de mettre en évidence l'organisation de la maitrise d'ouvrage et de la maitrise d'œuvre ${ }^{15}$. La maitrise d'ouvrage était assurée par la commune qui dirigea de manière effective le chantier, tâche dont les commis des échevins s'acquittèrent consciencieusement. Pour autant, les textes ne laissent pas planer le moindre doute quant au véritable commanditaire: «Comme ja pieça, par nostre tres doubté seigneur, monseigneur le duc de Berry [...] heust esté ordené un horloge estre faiz en sa dicte ville. $\aleph^{16}$. Même au plan technique, le mécène gardait la haute main sur l'entreprise car, si la maîtrise d'œuvre était confiée à des artisans locaux, les devis et certificats étaient délivrés par ses hommes: Guy de Dampmartin (général maître des œuvres du duc); Jean Guérart (lieutenant de Guy de Dampmartin); Robert Fouchier (maître des œuvres de charpenterie du duc) et Jean Macé (maître des œuvres du duc en Poitou). Enfin le système de financement était lui aussi verrouillé par le prince qui avait nommé le receveur chargé de collecter les sommes destinées à l'œuvre ${ }^{17}$. Le coût de l'entreprise équivalait à trois fois celui exigé par les travaux de fortification et de voierie ${ }^{18}$ . Jamais les finances ordinaires de la ville n'auraient été en mesure d'y pourvoir sans l'intervention de Jean de Berry qui subventionna le chantier par l'affectation de taxes et d'impôts ${ }^{19}$. Voici bien des éléments objectifs qui laisseraient supposer que l'horloge de Poitiers n'était que le projet du duc. Mais le Gros horloge semble au contraire une entreprise aux finalités partagées par les deux commanditaires. Par ce geste, Jean de Berry et les échevins scellaient durablement leur entente commune. Le mécène n'avait pas oublié la bonne volonté dont la ville avait fait preuve lors de la reconquête du Poitou sur les Anglais en 1372 (ill. 1). 
III. 1 Poitiers, le Gros horloge (plan de situation)

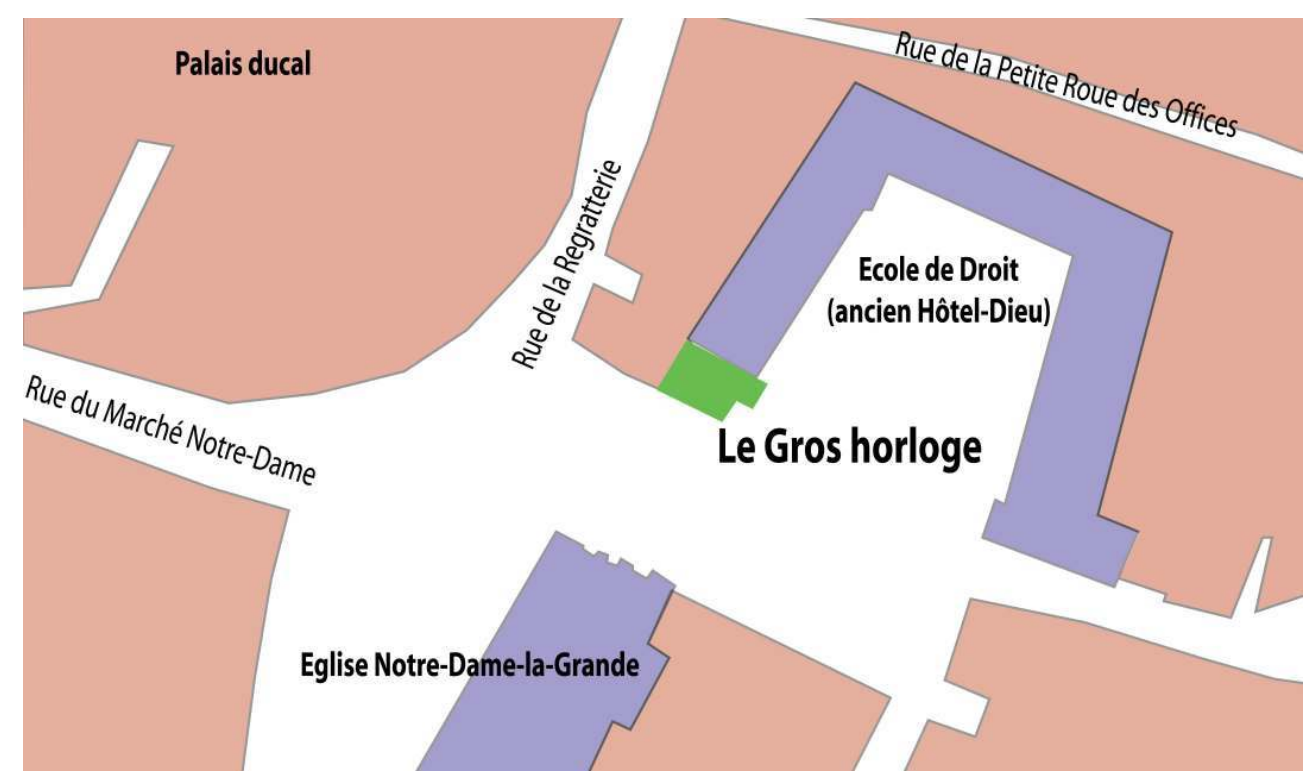

D’après le cadastre napoléonien, Arch. dép. Vienne, 4P1188, sect. L, feuille 2.

Thomas Rapin

Il fut décidé que le Gros horloge prendrait place au cœur de la vie citadine : face à NotreDame-la-Grande, dans les proches environs du marché et du palais ducal. L'édifice culminerait à 41 mètres au-dessus de l'entrée de l'aumônerie Notre-Dame-la-Grande (l'Hôtel-Dieu). Le projet architectural se résumait donc à la pose d'un beffroi sur une structure maçonnée préexistante. Celle-ci était de plan carré et comprenait au niveau du sol le passage vers l'aumônerie couvert par quatre voûtes d'ogives. Au premier étage était la chapelle Saint-Thomas communiquant de plain-pied avec le premier étage de la grande salle des malades. C'est aux niveaux supérieurs que l'on trouvait «la maison de l'horloge » contenue dans un beffroi charpenté couvert d'ardoises dont l'accès se faisait directement depuis la rue par l'intermédiaire un escalier en vis. "La maison de l'horloge" possédait une fenêtre ouverte sur Notre-Dame-la-Grande. Au-dessus de l'horloge, dans "l'aiguille", battaient les trois cloches du Gros horloge. Les cinq bannières de plomb qui couronnaient l'ouvrage - les angles portant les armes de la ville et au centre celles du duc - rappelaient que le Gros Horloge était le produit d'une volonté commune $^{20}$. Le monument connut une très honorable longévité. Il sonna les heures du jour et de la nuit ${ }^{21}$ aux Poitevins jusqu'en 1740. C'est seulement devant l'impossibilité de conserver l'édifice en l'état que la municipalité ordonna sa démolition qui prit fin sous la Restauration $^{22}$ (ill. 2) 


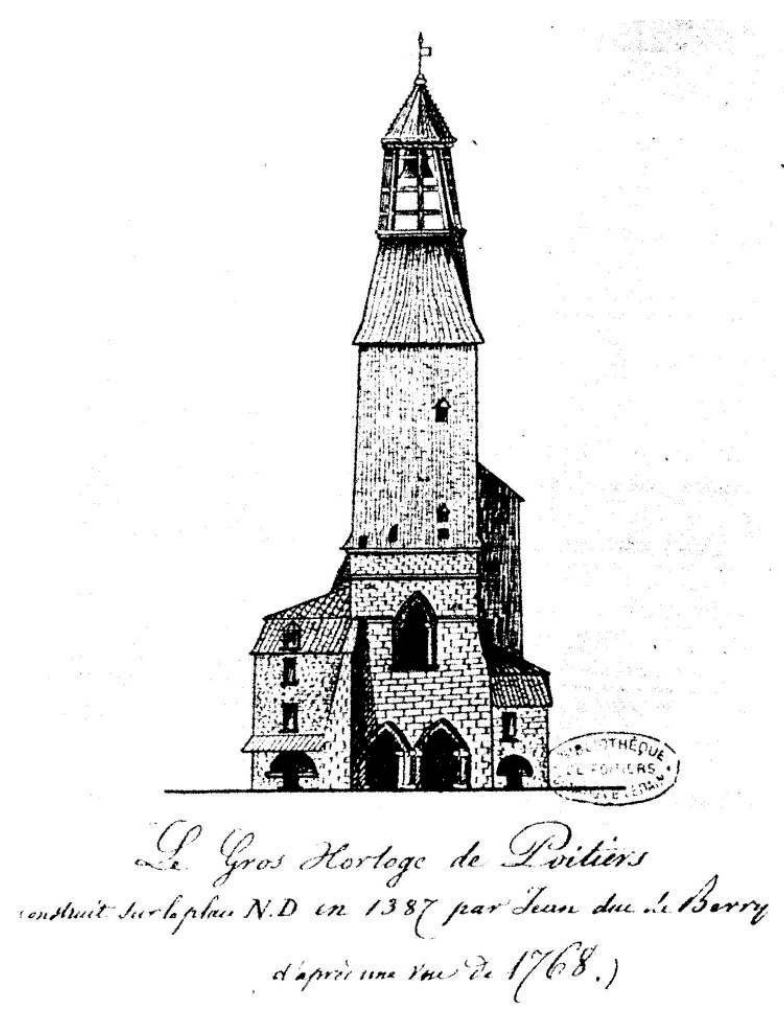

Anonyme, d'après un dessin réalisé vers 1768. Arch. mun. Poitiers.

Cl. Thomas Rapin

La construction s'étendit sur quatre années de 1385 à 1389 . On réquisitionna à cet effet les environs du chantier. Les cloches furent fondues dans le cimetière de Notre-Dame-laGrande. La maison d'un particulier, proche de l'aumônerie, fut louée pendant plus d'une année pour permettre aux couvreurs d'ardoises et aux plombiers de travailler ${ }^{23}$. Les centaines de pièces de parchemin conservées aux Archives municipales permettent d'entrer dans le détail du chantier ${ }^{24}$. Quatre importants marchés présidèrent à la réalisation de l'ouvrage. Les deux premiers, respectivement pour la charpenterie ${ }^{25}$ et la couverture $^{26}$, furent confiés par les échevins à des entreprises locales. L'approvisionnement en matériaux était sans commune mesure avec ce que nécessitaient d'ordinaire les travaux urbains et au premier chef le bois d'œuvre. Bien que Jean de Berry eût concédé à cet effet une part de la production du parc de Montreuil-Bonin et de la forêt du Coulombiers, la ville fut contrainte de demander une nouvelle concession forestière ${ }^{27}$. Le choix d'un essentage d'ardoise témoigne de la qualité architecturale souhaitée par les commanditaires. Poitiers n'était pas une ville d'ardoise qu'il fallait faire venir du val de Loire jusqu'à Châtellerault puis par chariot jusqu'à Poitiers.

Les deux autres marchés, celui de la fonte des cloches et celui du mécanisme d'horlogerie, relevèrent directement des sphères d'influences du duc de Berry avec les milieux artistiques à Paris. C'est dans la capitale, qu'il recruta le saintier Jean Haumont - ou Osmont $^{28}$ - qui vint à Poitiers dès le mois de juillet $1386^{29}$ afin d'y réaliser trois cloches pour le Gros horloge: une grande et deux petites ${ }^{30}$. La fonte de la grande cloche commença au printemps 1387, mais devant la difficulté de la ville à réunir la quantité de métal nécessaire, Jean de Berry fit réquisitionner les deux cloches de l'abbaye voisine de 
Saint-Cyprien laissant à la ville le soin de pourvoir ultérieurement à leur remplacement ${ }^{31}$. Le levage et la bénédiction de la grande cloche n'eurent lieu qu'après la Toussaint $1387^{32}$. En conséquence Jean Haumont, qui avait contracté un marché de $300 \mathrm{Et}^{33}$, réclama et obtint en juin 1387 un supplément de $30 £$ pour le nombre de jours chômés ${ }^{34}$. Moins de dix années après avoir été mise en service, la sonnerie connut une avarie ; il fallut la déposer à grands frais et refondre la grande cloche, opération confiée à un certain Guillaume Le Roux ${ }^{35}$.

17 Enfin le quatrième marché - ou plutôt engagement - fut celui qui lia la ville à son nouvel horloger Pierre Merlin « orlogeur de monseigneur le roy ». Il avait travaillé pour Charles VI à la «Grand Horloge » de la cathédrale de Sens (1376-1377) puis à celle d'Angers pour le compte du duc d'Anjou en 1387. Recruté directement par Jean de Berry, Pierre Merlin fut nommée gouverneur du Gros horloge dans une lettre signée de sa main et datée du 5 avril $1388^{36}$. Dans un rôle daté du 4 janvier précédent, on note une dépense pour la clef

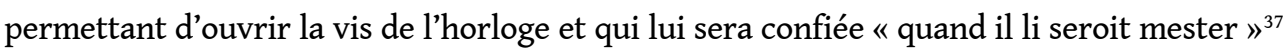
- Ainsi, avant son entrée en fonction, il devait certainement être occupé à préparer l'installation du mécanisme. D'ailleurs, dans sa lettre, Jean de Berry précisait que les émoluments de Pierre Merlin lui seront versés «a commencer le jour que ledit orloge sera prest, parachevé et bien sonnant ». Le 5 juillet suivant, deux charpentiers reçurent l'ordre de retirer des planchers pour installer l'horloge ${ }^{38}$. Une partie de ces travaux était directement dictée par Pierre Merlin qui avait la possibilité d'établir des devis et d'ordonner des travaux. Par exemple, en février et en avril 1389, il ordonnait aux charpentiers de renforcer le plancher " ou sont les roues $»^{39}$. La comptabilité municipale reste totalement muette au sujet de la réalisation du mécanisme horloger lui-même. On peut supposer que celui-ci avait été commandé et financé directement par le duc de Berry probablement par un marché comme celui contracté entre Pierre de Daimleville et la duchesse de Bourgogne pour le château de Nieppe.

Revenons maintenant aux attributions de Pierre Merlin précisées dans sa lettre de nomination. Il est question de gouverner l'horloge. À cette fin, la ville versera à l'horloger une pension annuelle de $100 £$ prises sur la " caisse des réparations de la ville » et versée en quatre termes. Sur ses gages, Pierre Merlin rémunérait également un certain Pierre Dorlain qui le secondait ${ }^{40}$. Cet engagement de la ville envers le gouverneur de l'horloge nommé par le prince rappelle le cas similaire du gouverneur de l'horloge du palais de la Cité dont les gages étaient payés par les Parisiens ${ }^{41}$. Pierre Merlin fut actif à Poitiers jusqu'en $1415^{42}$. Deux ans plus tard, les textes relèvent le nom de son successeur : Jean Chevalier ${ }^{43}$.

Le cas du Gros horloge nous conduit à repenser le corpus monumental de Jean de Berry. Les horloges publiques ont toute légitimité à être créditées à son mécénat. La maîtrise d'ouvrage avait beau être déléguée aux communes, il s'agissait bien de projets initiés par le prince. Malheureusement que ces programmes ont laissé derrière eux peu d'éléments à étudier et les trop rares documents iconographiques ont peu joué en leur faveur. Le beffroi de Poitiers n'a pas été jugé digne d'intérêt par les érudits locaux ${ }^{44}$. Curieusement la belle tour de l'horloge de Riom n'a pas non plus suscité l'intérêt des historiens de Jean de Berry. Pour autant la seule lecture des pièces fournies par le chantier du Gros horloge montre le haut degré de conception et de mise en œuvre exigés par de tels programmes dont les coûts s'avèrent en fait comparables à ceux des chantiers castraux ou palatiaux. Ils avaient assurément sur les citadins un impact tout aussi fort que les grandes constructions, plus que s'épandre dans l'espace et le paysage, les horloges occupaient le 
temps. Enfin n'oublions pas aussi la fascination des gens d'alors que le rouage mécanique qu'une force invisible mettait en mouvement. Beaucoup y virent la métaphore du bon gouvernement, de la sagesse divine, ou encore du désir comme Jean Froissart dans son Orloge amoureus ${ }^{45}$; d'autres dictons populaires furent moins élogieux «c'est l'orloge du palais, elle va comme il lui plait $»^{46}$.

\section{NOTES}

1. Thomas Rapin, Les chantiers de Jean de France duc de Berry: Maîtrise d'ouvrage et architecture à la fin $d u$ Moyen Age, thèse d'histoire de l'art, université de Poitiers, Claude Andrault-Schmitt et Jean Chapelot (dir.), 2010, 3 vol., 760 p.

2. Julie Singer, «L'horlogerie et la mécanique de l'allégorie chez Jean Froissart », Médiévales, t. 49, automne 2005, p 156.

3. Gerhard Dorhn-van Rossum, Histoire de l'heure, l'horlogerie et l'organisation du Temps, Paris, éditions de la maison des sciences et de l'homme, 1997, p. 136.

4. Ibid., p. 146.

5. Ibid., p. 143.

6. Paul Gauchery, «Le timbre de l'horloge du duc de Berry à la cathédrale de Bourges », Mémoires de la Société Historique, Littéraire et Scientifique du Cher, t. 28, 1914, p. 15-22.

1. Jean Chapelot, "La cloche de l'horloge de Charles V sur le châtelet du donjon ", Dossier d'Archéologie, n² 289, déc. 2003 - jan. 2004, p. 74.

2. Paul Gauchery, "Le timbre de l'horloge du duc de Berry...», op. cit., p. 15-22. Alfred de Champeaux et Paul Gauchery, Les travaux d'art exécutés pour Jean de France, duc de Berry, Paris, H. Champion, 1894, p. 194-195 ; Toulgoet-Treanna (comte de), « les comptes de l'hôtel du duc de Berry (1370-1413) », Mémoires de la Société des Antiquaires du Centre, t. 20, 1893-1894, p. 64 et suiv. ; Thomas Rapin, Les chantiers de Jean de France..., op. cit., note ${ }^{\circ} 1655$.

3. Emmanuel Poulle, "Instruments scientifiques et horlogerie ", in Art et société au XVe siècle, Christiane Pirgent (dir.), Paris, Maisonneuve \& Larose, 1999, p. 536-539.

4. Horloge de Vincennes (Jean Chapelot, «La cloche de l'horloge de Charles V... », op. cit., p. 75). Horloge de l'église Saint-Jacques-aux-Pélerins à Paris (Gerhard Dorhn-van Rossum, Histoire de l'heure..., op. cit., note 172).

1. Dany Sandron, «Les flèches de Strasbourg, Ulm, Vienne et Francfort: l'intervention municipale dans les grands chantiers d'église vers 1400 », Revue de l'art, n 166, 2009-4, p. 27-42.

2. Jean Chapelot, «La cloche de l'horloge de Charles V... », op. cit., p. 74.

3. Gerhard Dorhn-van Rossum, Histoire de l'heure..., op. cit., p. 142.

4. Ibid., p. 164-165.

5. Josiane Teyssot, Riom, capitale et bonne ville d'Auvergne, 1212-1557, Nonette, Créer, 1999, p. 114. La tour est toujours en place aujourd'hui ( $n^{\circ} 5$, rue de L'horloge) mais le mécanisme d'origine est inconnu car il fut remplacé par un autre dès le XVI (Ibid., p. 203).

6. Robert Favereau et Jean-Yves Riou, « Niort au Moyen Age » dans Histoire de Niort, des origines à nos jours, Poitiers, Pillard G, 1987, p. 85. Sainte-Hermine (de), «Recherches sur l'ancien hôtel de ville de Niort », Revue Anglo-Française, t. IV, 1836, p. 446-447.

7. Robert Gauchery, «Le château de Mehun-sur-Yèvre», Congrès Archéologique de France, Bourges, t. 94, 1931, p. 345. 
8. L. Duret, «La tour de la grosse horloge de Saint-Jean-d'Angély », Recueil de la Commision des Arts et Monuments Historiques de la Charente inférieure, t. 12, 1893-1894, p. 102-107.

9. Gerhard Dorhn-van Rossum, Histoire de l'heure..., op. cit., p. 142.

10. Paul Gauchery, «Le timbre de l'horloge du duc de Berry... », op. cit., p. 16.

11. Inscription de la cloche de Bourges rapportée dans les Opuscules de Nicolas Catherinot a été corrigée par Louis Raynal (Histoire du Berry, Paris, Guénégaud (rééd.), 1972, t. II, p. 403) : Metropoli datum Biturie sit me poli prefecit cursib(u)s dux Iohannes, comes pictanie que horarii preco sim civib(u)s l'an mil CCC LXXII « Le duc Jean, comte de Poitou, m'a donnée à la métropole de Bourges et ainsi préposée au cours du temps afin que je sois pour les citoyens le héraut de l'horloge. L'an $1372 »$.

12. Paul Gauchery, "Le timbre de l'horloge du duc de Berry... », op. cit., p. 15-22: " pour un reloige que mon dit seigneur a ordonné estre fait a Bourges » 400 francs, quittance du 26 décembre (Lehoux Françoise, op. cit., t. I, p. 273, note 5).

13. Arch. dép., Puy-de-Dôme, $5 \mathrm{C} 179$, fol. $4 v^{\circ}$ et 7.

14. Thomas Rapin, Les chantiers de Jean de France..., op. cit., p. 349-361.

15. Arch. mun., Poitiers, 27 J, n 189-358 (1380-1390) édité dans Catherine Provost, Les travaux communaux dans la ville de Poitiers à la fin du XIV e siècle, Mémoires de maîtrise d'histoire médiévale, université de Poitiers, Cécile Treffort et Régis Recht (dir.), 2003, vol. 2, 342 p. Arch. mun., Poitiers, $25 \mathrm{~J} \mathrm{n}^{\circ} 61-63$ (1394), $27 \mathrm{~J}$ 367-399 (1396-1399), $26 \mathrm{~J}, \mathrm{n}^{\circ} 100-120$ (1397), n¹21-150 (1398-1399), $28 \mathrm{~J}, \mathrm{n}^{\circ}$ 438-515 (1407-1408) et $29 \mathrm{~J}, \mathrm{n}^{\circ} 542-545$ (1416-1417).

16. Ibid., 27 J, n² 292 (10 juin 1392)

17. Ibid.

18. Durant la décennie 1380, le coût total de l'entreprise s'élevait à $3151 £ 1 \mathrm{~s} 3 \mathrm{~d}$ contre $979 £ 12$ s. 11d. (Catherine Provost, Les travaux communaux. op. cit., tableau $\mathrm{n}^{\circ} 30$ ).

19. Arch. mun., Poitiers, $27 \mathrm{~J}, \mathrm{n}^{\circ} 220$.

20. Ibid., $\mathrm{n}^{\circ} 279-281$ et 300 .

21. Inscription relevée avant la destruction du Gros Horloge en 1804 et publié par Paul Gauchery («Le timbre de l'horloge du duc de Berry...», op. cit., p. 17). Hanc campanam cum horlogio ad notificandum horas diei et noctis fecit fieri inclitissimus princeps Joannes [...] , ad laudem Christi et civium suorum, quam contstruxit Guillemus de Roucy habitor ville Cantantis, anno domini millesimo trecentesimo nonagesimo sexto.

22. Pierre-Eugène Pilotelle, «Le Gros Horloge de Poitiers », Bulletin de la Société des Antiquaires de l'Ouest, t. 4, 1844-1846, p. 221-238.

23. Arch. mun., Poitiers, $27 \mathrm{~J}, \mathrm{n}^{\circ} 355$ (de mai 1387 à août 1388).

24. Provost Catherine, Les Travaux communaux..., vol. 1, p. 28-45. et Pilotelle Pierre-Eugène, « Le Gros Horloge... », op. cit., p. 409-421).

25. Arch. mun., Poitiers, $27 \mathrm{~J}, \mathrm{n}^{\circ}$ 208, 214, 229, 251, 268 (marché conclu pour un montant de $620 £$ t., février 1387 - septembre 1387).

26. Ibid., $\mathrm{n}^{\circ}$ 238, 253, 296, 322, 334 (marché de $550 £ \mathrm{t}$. contracté avec Guy de Dampmartin, général maître des œuvres de Jean de Berry le 20 mai 1387. Les travaux eurent lieu du mois d'août 1387 à janvier 1389).

27. Ibid., $\mathrm{n}^{\circ}$ 205-206.

28. Ibid., $\mathrm{n}^{\circ} 244$.

29. Ibid., $\mathrm{n}^{\circ} 242$.

30. Ibid., $\mathrm{n}^{\circ}$ 339. Les heures étaient sonnées d'ordinaire sur la grande (Ibid., $26 \mathrm{~J}, \mathrm{n}^{\circ} 137$ ). Le rôle exact des deux autres cloches est mal cerné. On sait néanmoins que la voûte de la chapelle SaintThomas était équipée d'une cloche (Pierre Rambaud, "L'assistance publique à Poitiers jusqu'à l'an V », Mémoires de la Société des Antiquaires de l'Ouest, t. VII, 1913, p. 8).

31. Ibid., $27 \mathrm{~J}, \mathrm{n}^{\circ} 241-242$.

32. Ibid., $\mathrm{n}^{\circ} 278$. 
33. Ibid., $\mathrm{n}^{\circ} 242$ et 244 .

34. Ibid., $\mathrm{n}^{\circ} 243$.

35. Catherine Provost, Les Travaux communaux..., op. cit., vol. 1, p.225 (année 1396).

36. Arch. mun., Poitiers, $27 \mathrm{~J} \mathrm{n}^{\circ} 293$.

37. Ibid., $\mathrm{n}^{\circ} 278$.

38. Ibid., $\mathrm{n}^{\circ} 314$.

39. Ibid., $\mathrm{n}^{\circ} 347$.

40. Ibid., n³57 et 358 (années 1390 et 1392)

41. Jean Guérout, "L'hôtel du roi au palais de la Cité à Paris sous Jean II et Charles V », dans Vincennes aux origines de l'État moderne, Jean Chapelot et Elisabeth Lalou (dir.), Paris, Presses de l'École normale supérieure, 1996, p. 236.

42. Arch. mun., Poitiers, $25 \mathrm{~J}, \mathrm{n}^{\circ} 61-63$.

43. Arch. mun., Poitiers, 29 J, n 563.

44. René Crozet, "Les constructions civiles d'intérêt public à Poitiers au Moyen Age ", dans Les Constructions civiles d'intérêt public dans les villes d'Europe au Moyen Âge et sous l'Ancien Régime et leur financement, Bruxelles, Pro Civitate, 1971, p. 45.

45. Julie Singer, «L'horlogerie et la mécanique... », op. cit., p. 155-172.

46. M. Chassant, " Notice historique sur la tour de l'horloge d'Évreux », Recueils des travaux de la société libre d'Agriculture, Science, Arts et Belles-lettres du département de l'Eure, t. IV, 1843, p. 283-316.

\section{RÉSUMÉS}

Dans la France du bas Moyen Age, l'horlogerie restait une technologie au service de la grande aristocratie qui protégeait alors les rares horlogers comme elle le faisait avec ses architectes, peintres et sculpteurs. Le grand mécène Jean de Berry (1340-1416) participa lui aussi à ce phénomène et notamment par la multiplication des tours d'horloge en place publique. Beaucoup reste à dire sur cet aspect méconnu de sa contribution à l'art. À travers les cas bien documentés des deux horloges publiques qu'il installa à Bourges et Poitiers, nous sommes en mesure de percevoir - au-delà de la prouesse technique - toute la portée sociale et politique de ces programmes conjuguant les volontés communes de Jean de Berry et communautés urbaines.

In France during the late middle ages, horlogery was still a technology for the high aristocrats which protected the rare watchmakers as they did with their architects, painters and sculptors. The great patron Jean de Berry (1340-1416) also took part in this kind of programs and especially the multiplication of clock towers in the public yard. Much could be said about this little known aspect of his contribution to art. Throught tow examples well documened of clock towers that he built in Bourges and Poitiers, we are able to perceive - beyond the technical prowess - all the social and political impact of these programs witch combines the common wishes of Jean de Berry and urban communities.

Im Spätmittelalter ist die Uhrenfabrik in Frankreich eine Technologie, wobei die Aristokratie eine wichtige Rolle spielte. Dadurch wurden die Uhrmacher, ähnlich zu den Architekten, den Malern und den Bildhauern unterestützt. Der berühmte Jean de Berry ließ auch viele öffentliche Turmuhren errichten. Vieles ist noch im Hinblick auf diesen unbekannten Aspekt seiner Aufträge zu erforschen. Zwei gut dokumentierte Beispiele aus Bourges und aus Poitiers veranschaulichen 
nicht nur die technische Spitzenleistung von Turmuhren, sondern auch die gesellschaftliche und politische Bedeutung dieser Werke, die sowohl für Jean de Berry, als auch für die städtischen Gemeinschaften von Interesse waren.

\section{AUTEUR}

\section{THOMAS RAPIN}

Né le 28 décembre 1971, Thomas Rapin est titulaire d'un doctorat en histoire de l'art obtenu en 2010 sous la direction de Claude Andrauld-Schmitt, centre d'Études supérieures de la civilisation médiévale, université de Poitiers, cotutelle avec Jean Chapelot, CNRS : Les chantiers de Jean de France, duc de Berry : architecture et maîtrise d'ouvrage à la fin du Moyen Âge. Il est l'auteur de plusieurs articles portant sur le mécénat de Jean de Berry ainsi que sur le problème de la commande architecturale et sur la production monumentale religieuse et civile à la fin du Moyen Âge. thomasrapin@hotmail.com 